\title{
IgE and TGF- $\beta$ Signaling: From Immune to Cardiac Remodeling
}

\author{
Hua Cao (iD ${ }^{\prime}$ \\ Chungang Xiao' \\ Zhangxiu $\mathrm{He}^{2}$ \\ Hong Huang $\mathbb{D D}^{3,4}$ \\ Huifang Tang ${ }^{1,4}$ \\ 'The First Affiliated Hospital, \\ Department of Cardiology, Hengyang \\ Medical School, University of South \\ China, Hengyang, Hunan, 42I00I. \\ People's Republic of China; ${ }^{2}$ The First \\ Affiliated Hospital, Department of \\ Nephrology, Hengyang Medical School, \\ University of South China, Hengyang, \\ Hunan, 42100I, People's Republic of \\ China; ${ }^{3}$ The First Affiliated Hospital, \\ Institute of Clinical Medicine, Hengyang \\ Medical School, University of South \\ China, Hengyang, Hunan, 42I00I, \\ People's Republic of China; ${ }^{4}$ The First \\ Affiliated Hospital, Institute of \\ Cardiovascular Disease, Hengyang \\ Medical School, University of South \\ China, Hengyang, Hunan, 42100I, \\ People's Republic of China
}

Correspondence: Hong Huang; Huifang Tang

The First Affiliated Hospital, Institute of Cardiovascular Disease, Hengyang Medical School, University of South China, Hengyang, Hunan, 42100I,

People's Republic of China

Email travel@126.com;

tanghuifang999@163.com

\begin{abstract}
Cardiac remodeling is accompanied by cardiac hypertrophy, fibrosis, and dysfunction, eventually leading to heart failure (HF). However, the molecular mechanisms involved in cardiac remodeling are complicated, especially the association with immune. Immunoglobulin E ( $\mathrm{IgE}$ ) is a class of immunoglobulins involved in immune response to specific allergens. Recently, Zhao et al characterized a novel specific role of $\operatorname{IgE}$ and its high affinity receptor (FceR1) in directly promoting pathological myocardial remodeling and cardiac dysfunction. Additionally, upon blocking IgE-FceR1 signaling using FceR1 genetic depletion or by administrating the anti-IgE monoclonal antibody omalizumab (Oma) in mice, they observed that cardiac hypertrophy and cardiac interstitial fibrosis induced by angiotensin II (Ang II) or transverse aortic constriction (TAC) were significantly suppressed. In contrast, IgE administration alone can aggravate pathological cardiac remodeling and dysfunction. RNA-seq and downstream analysis indicated that TGF- $\beta$ was the common pathway and the most pivotal mediator in IgE-FceR1-induced cardiac remodeling and dysfunction. Furthermore, the administration of a TGF- $\beta$ inhibitor could ameliorate cardiac remodeling and improve cardiac function. Therefore, these findings suggest that IgE-FceR1 maybe promising therapeutic targets for cardiac remodeling and provide an experimental basis for the use of omalizumab for HF patients combined with high serum IgE levels or allergic diseases.
\end{abstract}

Keywords: cardiac remodeling, IgE- FceR1, immunoglobulins, TGF- $\beta$

Pathological cardiac remodeling is an adaptive response to cardiac stress, accompanied by myocardial hypertrophy, interstitial fibrosis, cell death, cardiac dysfunction, and eventually leading to heart failure (HF). ${ }^{1}$ Immunoglobulins, as major host proteins in plasma, function as anti-bacterial and anti-viral agents by strengthening cell phagocytosis. Immunoglobulin E (IgE) is a class of immunoglobulins involved in immune response to specific allergens. ${ }^{2,3}$ They play a central role in the development and manifestation of allergic reactions. ${ }^{4}$ Functionally, allergen-induced crosslinking of Fe\&R1-bound IgE activates basophils and mast cells, followed by degranulation. The degranulation of basophils and mast cells results in the liberation of proinflammatory and vasoactive mediators, inducing classical symptoms of an allergic reaction. ${ }^{5}$ Allergic diseases, including immediate hypersensitivity reactions, anaphylaxis, allergic asthma, atopic dermatitis, chronic spontaneous urticaria (CSU), etc. are primarily mediated by the IgE-Fc\&R1 signaling pathway. A previous study indicated that total serum IgE levels before anti-IgE antibodies intervention correlate negatively with the time-to-relapse in patients with $\mathrm{CSU},{ }^{6}$ which is consistent with the pathological molecular mechanism of the IgE-FceR1 signaling 
pathway. However, IgE-FceR1 also critically contributes to host defense against parasites and venoms. ${ }^{7}$

Although much focus regarding the biological properties of $\mathrm{IgE}$ is centered around immune response to specific allergens, increasing evidence has uncovered unexpected pathological roles of $\operatorname{IgE}$ in the cardiovascular system. A population-based cohort study suggested that the elevation of serum IgE levels may be a risk factor for increased cardiovascular mortality. ${ }^{8}$ Existing literature also revealed that IgE and the FceR1 $\alpha$ were present in human atherosclerotic lesions, which are localized particularly in macrophage-rich areas. $\mathrm{IgE}$ deficiency in $\mathrm{ApoE}^{-/-}$mice reduced atherosclerosis by decreasing the lesion macrophage content and inflammation. ${ }^{9}$ In addition, blocking the IgEFceR1 axis reduced inflammation and apoptosis both in atherosclerotic plaques and abdominal aortic aneurysms (AAAs). ${ }^{9,10}$ However, whether IgE-FceR1 signaling is involved in cardiac remodeling remains elusive.

A recent study in Circulation by Zhao et al identifies IgE as a novel promoter of cardiac remodeling in mice and humans, which functions in an IgE-FceR1-dependent manner. ${ }^{11}$ Statistical analysis of 4671 participants from the National Health and Nutrition Examination Survey (NHANES) showed a strong positive relationship between serum IgE and NT-pro BNP in HF patients. In concordance with the HF patients, high serum IgE levels and cardiac FceR1 expression were observed in mice subjected to transverse aortic constriction (TAC) or angiotensin II (Ang II). In addition, upon blocking IgE-FceR1 signaling using FceR1 genetic depletion or administrating the anti-IgE monoclonal antibody omalizumab (Oma) in mice, cardiac hypertrophy and cardiac interstitial fibrosis induced by Ang II or TAC were significantly suppressed. In contrast, IgE administration alone can aggravate pathological cardiac remodeling and dysfunction. Moreover, chimeric mice generated by bone marrow transplantation (BMT) demonstrated that the effect of $\operatorname{IgE}$ on Ang II-induced cardiac remodeling was not mediated by bone marrow-derived cells. To further determine whether IgE can directly function in cardiomyocytes (CMs) and cardiac fibroblasts (CFs), they first verified that FceR1 was expressed in CMs and CFs. In addition, IgEinduced hypertrophy, CFs activation and matrix protein production were remarkably alleviated by FceR1 knockdown. All above suggested that IgE directly promoted cardiac remodeling in an FceR1-dependent manner in CMs and CFs.

To explore the potential mechanisms of the IgE-FceR1 axis, RNA-seq was performed on both CMs and CFs treated with or without IgE. The transcriptomes profiling showed reduced expression of TGF- $\beta$ and its downstream signaling molecules, including PI3K, AKT, pSmad1/5/9, and pSmad $2 /$ 3, in the hearts of FceR1-KO mice compared to WT mice after Ang II infusion. Similar effects were demonstrated in omalizumab-treated mice. However, TGF- $\beta$ inhibitor SB431542 significantly alleviated CM hypertrophy and CF activation induced by IgE treatment. In summary, TGF- $\beta$ is likely to be the most pivotal mediator in IgE-FceR1-induced cardiac remodeling and dysfunction (Figure 1).

As it often happens, this study opens a range of interesting questions. First, existing literature suggested that rituximab (RTX), an antibody against CD20, can reverse cardiac remodeling and improve cardiac function through inhibiting Th2 cytokine-mediated IgG production from B cells. ${ }^{12}$ A marked increase in B cell accumulation in vasculature was observed in Zhao's work, which may account for the elevation of serum IgE. As we known, IgE is the prototypical antibody class produced during type 2 immune responses, which are induced during tissue remodeling. ${ }^{13-15}$ Although the role of $\operatorname{IgE}$ in promoting cardiac remodeling has been just reported, whether there is an increased level of IgG with the accumulation of $\mathrm{B}$ cells and how it functions during this process remains to be clarified. Second, can

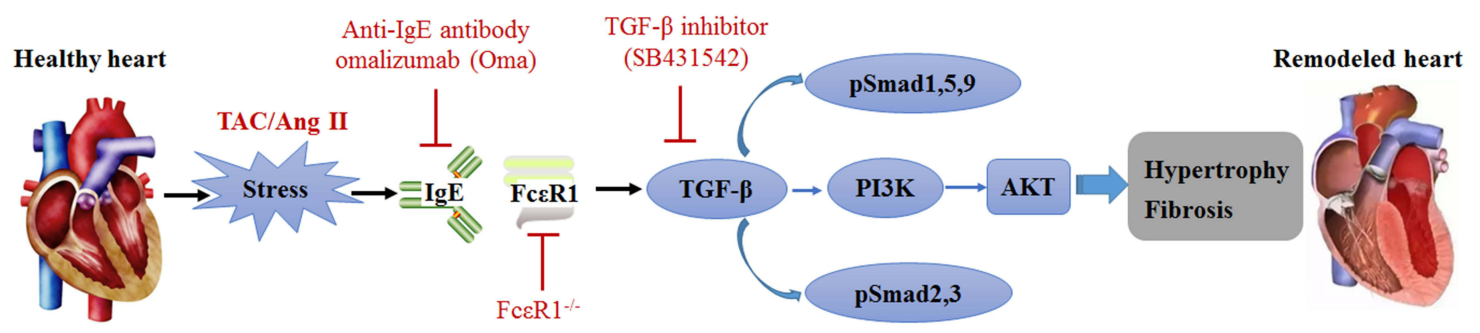

Figure I Proposed model for the role of IgE-FceRI signaling in cardiac remodeling. Activated TGF- $\beta /$ PI3K-AKT and TGF- $\beta /$ Smads signaling after serum IgE and cardiac FceR I expression induced by stress (TAC, Ang II) in both cardiomyocytes and cardiac fibroblasts, which ultimately contributes to cardiac pathological remodeling. TGF- $\beta$ is likely to be a critical mediator for IgE-FceRI in promoting cardiac hypertrophy and fibrosis. Suppression of the IgE-FceRI signaling using FceRI-KO mice or anti-lgE antibodies (omalizumab) significantly ameliorates cardiac remodeling in mice. 
elevated $\operatorname{IgE}$, locally and in circulation, influence the cardiac microenvironment or activate other cardiac resident cells, such as endothelial cells and macrophages? What's the contributions during the development of cardiac remodeling? Finally, regarding the specific mechanisms underlying cardiac cells hypertrophy and cardiac fibroblast activation, Zhao et al have demonstrated the crucial role of TGF- $\beta$ signaling in this process. However, bioinformatic analyses show that other pathways, such as the $\beta$-AR signaling pathway, chemokine-related pathway, and cytokine-mediated inflammation, are also significantly changed in this process. According to the reports describing cross-talk and mutual regulation between $\beta$-AR and TGF- $\beta$ signaling, ${ }^{16}$ we wonder that whether $\beta$-AR contributes to TGF- $\beta$ activation and regulates cardiac hypertrophy via activation of the cAMP-PKA pathway in cardiomyocytes? Additionally, previous studies have suggested that FceR2, the low affinity IgE receptor, may contribute to the production of chemokines, ${ }^{17}$ that have also been shown to function directly in cardiac remodeling. ${ }^{18}$ Therefore, we speculate that elevated IgE induces the expression of FceR2 in cardiomyocytes and then activates the chemokine-related pathway. The expression of FceR2 in cardiomyocytes and its contact with chemokine-related pathway are worth being discussing. Nevertheless, further study should be conducted to address the issues mentioned above and identify more effective therapeutic targets for HF.

In summary, Zhao et al demonstrate the potential implication of high serum $\operatorname{IgE}$ to pathological cardiac remodeling. Targeting the IgE-FceR 1 axis via the activation of TGF- $\beta$ signaling in the heart, which highlights how immunoglobulins affect cardiac functions other than inflammation and immunity. In addition, the application of omalizumab, a clinical IgE antagonist, provides important preclinical evidence for the treatment of HF patients combined with allergic reactions. A recent study by their team has verified that elevated serum IgE promotes pathological cardiac fibrosis by modulating miR-486a-5p and downstream factor Smad1. ${ }^{19}$ These findings have broadened our horizons for the upstream regulators of the IgE-FceR 1 signaling pathway. Although the specific mechanisms were not completely elucidated, the work of Zhao et al has further clarified the linkages between immunity and cardiac remodeling. To the best of our knowledge, this is the first study to elucidate the association between serum IgE levels and cardiac remodeling. Overall, IgEFceR1 may be promising novel therapeutic targets for the treatment of cardiac remodeling, with high translational potential, especially for patients with HF combined with high serum IgE levels or allergic diseases.

\section{Funding}

This work was supported by the National Natural Science Foundation of China (81900678) and the Natural Science Foundation of Hunan Province (2019JJ50555), the Open Project Program of Guangxi Key Laboratory of Centre of Diabetic Systems Medicine, Guilin Medical University (GKLCDSM-20200101-03), the Hunan Province Technology Innovation Guidance Program-Clinical Medical Technology Innovation Guidance Project (2018SK51603), and the Science Foundation of The Health and Family Planning Commission in Hunan Province of China (B2019122, No. 20201920).

\section{Disclosure}

The authors report no conflicts of interest in this work.

\section{References}

1. Nakamura M, Sadoshima J. Mechanisms of physiological and pathological cardiac hypertrophy. Nat Rev Cardiol. 2018;15(7):387-407. doi:10.1038/s41569-018-0007-y

2. Maurer M, Altrichter S, Schmetzer O, Scheffel J, Church MK, Metz M. Immunoglobulin E-mediated autoimmunity. Front Immunol. 2018;9:689. doi:10.3389/fimmu.2018.00689

3. Tanaka S, Furuta K. Roles of IgE and histamine in mast cell maturation. Cells. 2021;10(8):2170. doi:10.3390/cells10082170

4. Gould HJ, Sutton BJ. IgE in allergy and asthma today. Nat Rev Immunol. 2008;8(3):205-217. doi:10.1038/nri2273

5. Galli SJ, Tsai M, Piliponsky AM. The development of allergic inflammation. Nature. 2008;454(7203):445-454. doi:10.1038/ nature 07204

6. Ertas R, Ozyurt K, Ozlu E, et al. Increased IgE levels are linked to faster relapse in patients with omalizumab-discontinued chronic spontaneous urticaria. $J$ Allergy Clin Immunol. 2017;140 (6):1749-1751. doi:10.1016/j.jaci.2017.08.007

7. Balbino B, Conde E, Marichal T, Starkl P, Reber LL. Approaches to target IgE antibodies in allergic diseases. Pharmacol Ther. 2018;191:50-64. doi:10.1016/j.pharmthera.2018.05.015

8. Min KB, Min JY. Risk of cardiovascular mortality in relation to increased total serum IgE levels in older adults: a population-based cohort study. Int J Environ Res Public Health. 2019;16(22):4350. doi:10.3390/ijerph 16224350

9. Zhang X, Li J, Luo S, et al. IgE contributes to atherosclerosis and obesity by affecting macrophage polarization, macrophage protein network, and foam cell formation. Arterioscler Thromb Vasc Biol. 2020;40(3):597-610. doi:10.1161/ATVBAHA.119.313744

10. Wang J, Lindholt JS, Sukhova GK, et al. IgE actions on CD4+ $\mathrm{T}$ cells, mast cells, and macrophages participate in the pathogenesis of experimental abdominal aortic aneurysms. EMBO Mol Med. 2014;6(7):952-969. doi:10.15252/emmm.201303811 
11. Zhao H, Yang H, Geng C, et al. Role of IgE-FecR1 in pathological cardiac remodeling and dysfunction. Circulation. 2021;143 (10):1014-1030. doi:10.1161/circulationaha.120.047852

12. Ma XL, Lin QY, Wang L, Xie X, Zhang YL, Li HH. Rituximab prevents and reverses cardiac remodeling by depressing B cell function in mice. Biomed Pharmacother. 2019;114:108804. doi:10.1016/j. biopha.2019.108804

13. Starkl P, Watzenboeck ML, Popov LM, et al. IgE effector mechanisms, in concert with mast cells, contribute to acquired host defense against staphylococcusaureus. Immunity. 2020;53(4):793-804.e9. doi:10.1016/j.immuni.2020.08.002

14. Wei L. Immunological aspect of cardiac remodeling: t lymphocyte subsets in inflammation-mediated cardiac fibrosis. Exp Mol Pathol. 2011;90(1):74-78. doi:10.1016/j.yexmp.2010.10.004

15. Yu Q, Horak K, Larson DF. Role of T lymphocytes in hypertension-induced cardiac extracellular matrix remodeling. Hypertension. 2006;48(1):98-104. doi:10.1161/01.HYP.0000227247.27111.b2
16. Leask A. Potential therapeutic targets for cardiac fibrosis: tGFbeta, angiotensin, endothelin, CCN2, and PDGF, partners in fibroblast activation. Circ Res. 2010;106(11):1675-1680. doi:10.1161/ CIRCRESAHA.110.217737

17. Ando T, Kitaura J. Tuning IgE: IgE-associating molecules and their effects on IgE-dependent mast cell reactions. Cells. 2021;10(7):1697. doi:10.3390/cells 10071697

18. Li Y, Zhang D, Kong L, et al. Aldolase promotes the development of cardiac hypertrophy by targeting AMPK signaling. Exp Cell Res. 2018;370(1):78-86. doi:10.1016/j.yexcr.2018.06.009

19. Zhao H, Yang H, Geng C, et al. Elevated $\mathrm{IgE}$ promotes cardiac fibrosis by suppressing miR-486a-5p. Theranostics. 2021;11 (15):7600-7615. doi:10.7150/thno.47845

\section{Publish your work in this journal}

The Journal of Inflammation Research is an international, peerreviewed open-access journal that welcomes laboratory and clinical findings on the molecular basis, cell biology and pharmacology of inflammation including original research, reviews, symposium reports, hypothesis formation and commentaries on: acute/chronic inflammation; mediators of inflammation; cellular processes; molecular mechanisms; pharmacology and novel anti-inflammatory drugs; clinical conditions involving inflammation. The manuscript management system is completely online and includes a very quick and fair peerreview system. Visit http://www.dovepress.com/testimonials.php to read real quotes from published authors. 